\title{
Thrombocytopenic syndromes in pregnancy
}

\author{
Matthew Yan', Ann K Malinowski ${ }^{2}$ and Nadine Shehata ${ }^{1,3}$
}

\begin{abstract}
The physiological changes in pregnancy result in platelet counts that are lower than in nonpregnant women. Consequently, thrombocytopenia is a common finding occurring in $7-12 \%$ of pregnant women. Gestational thrombocytopenia, the most common cause of low platelet counts, tends to be mild in most women and does not affect maternal, fetal or neonatal outcomes. Gestational thrombocytopenia needs to be distinguished from other less common causes of isolated thrombocytopenia, such as immune thrombocytopenia, which affects approximately $3 \%$ of thrombocytopenic pregnant women and can lead to neonatal thrombocytopenia. Hypertensive disorders of pregnancy and thrombotic microangiopathies are both associated with thrombocytopenia. They share a considerable number of similar characteristics and are associated with significant maternal and neonatal morbidity and rarely mortality. Accurate identification of the aetiology of thrombocytopenia and appropriate management are integral to optimizing the pregnancy, delivery and neonatal outcomes of this population. Clinical cases are described to illustrate the various aetiologies of thrombocytopenia in pregnancy and their treatment.
\end{abstract}

\section{Keywords}

Thrombocytopenia, pregnancy, immune thrombocytopenia, pre-eclampsia, thrombotic microangiopathy

Date received: 8 April 2015; accepted: 29 July 2015

\section{Case I}

A 31-year-old gravida 1 para 0 at 24 weeks' gestation has a platelet count of $82 \times 10^{9} / \mathrm{L}$. She does not have a personal history or family history of thrombocytopenia. She has never had symptoms suggestive of an underlying bleeding tendency. The current pregnancy has been uncomplicated. She is normotensive, and urinalysis does not demonstrate proteinuria. She does not have other cytopenias. Liver enzymes and serum creatinine are within the normal range.

The normal range for platelet counts is $10 \%$ lower in pregnancy than in nonpregnant women because of haemodilution and increased platelet activation and clearance. ${ }^{1-3}$ A platelet count of $<150 \times 10^{9} / \mathrm{L}$ occurs in $7-12 \%$ of the pregnant population. ${ }^{3,4}$ However, only $1 \%$ of pregnant women have levels $<100 \times 10^{9} / \mathrm{L}^{5}$ Approximately $75 \%$ of all individuals with thrombocytopenia in pregnancy have gestational thrombocytopenia (GT) or incidental thrombocytopenia of pregnancy. Of these, $15 \%$ have hypertensive syndromes, and 3-4\% have immune thrombocytopenia (ITP). ${ }^{5}$

\section{Isolated thrombocytopenias}

Gestational thrombocytopenia. GT is diagnosed by a reduction in the platelet count in the absence of other clinical, haematological and biochemical changes. With GT, a low platelet count is commonly observed during the second or third trimester, with a nadir platelet count generally greater than $70 \times 10^{9} / \mathrm{L}$, although there are reports of women having lower platelet counts. ${ }^{6}$ Resolution of thrombocytopenia is prompt, usually within days to two months postpartum. ${ }^{7}$ This maternal thrombocytopenia is not associated with fetal thrombocytopenia. ${ }^{5}$

Other causes of isolated thrombocytopenia include pseudothrombocytopenia, viral-associated thrombocytopenia, e.g. hepatitis B virus, hepatitis $\mathrm{C}$ virus, human immune deficiency virus, hereditary thrombocytopenia (e.g. Type IIb von Willebrand's disease), vitamin B12/folate deficiency, hypersplenism, drugs, and ITP. These may be differentiated by history, e.g. if there is a family history of a haemorrhagic tendency, examination, laboratory investigations and/or medical imaging (Table 1).

Other laboratory investigations such as screening for antiphospholipid antibodies (aPLs) in patients with thrombocytopenia is debateable and has been advised only when there is suspicion of antiphospholipid antibody syndrome (APS). ${ }^{2}$ The rationale for not screening for aPL is because only a small proportion will likely have
APS. Previous reports have overestimated the frequency of aPL in patients with thrombocytopenia because of the lack of adherence to criteria to diagnose aPL (e.g. anticaridiolipin immunoglobulin (Ig) G or IgM levels $>40$ GPL or MPL, respectively, or $>99$ th percentile 12 weeks apart). ${ }^{8}$ In addition, the risk of thrombosis in individuals with ITP has not definitively been shown to correlate with the presence/ absence of aPL. ${ }^{9}$ In contrast, screening for aPL has been advocated as thrombocytopenia occurs in $10-20 \%$ patients with aPL, ${ }^{10,11}$ and there is potentially a risk of thrombosis in asymptomatic carriers of aPL (5\%/year). ${ }^{12}$ Accordingly, a careful selection of pregnant patients with thrombocytopenia to screen for aPL, i.e. ITP with a prior fetal loss and the use of established criteria to identify aPL may reduce the incorrect classification of women as having aPL and identify women at higher risk of thrombosis.

Pseudothrombocytopenia or spurious thrombocytopenia occurs in $0.1-0.2 \%$ of the population and is secondary to platelet agglutination induced by ethylenediaminetetraacetic acid (EDTA), a common anticoagulant used in collection tubes for haematological tests. ${ }^{13}$ Platelet clumping results in the inability of haematology analysers to estimate an accurate platelet number. Pseudothrombocytopenia is not associated with an increased risk of bleeding, as the platelet number is not reduced. Determining the platelet count in collection tubes using citrate as an anticoagulant, instead of EDTA, will usually provide a more accurate platelet number; however, platelet clumping may persist in $15-20 \%$ of samples. ${ }^{13}$

Primary ITP may be difficult to distinguish from GT. Figure 1 highlights the characteristics that differentiate gestational from primary ITP. If the diagnosis is unclear at the time of delivery, intrapartum management should reflect the treatment for ITP.

'Department of Medicine, Division of Hematology, University of Toronto, Toronto, Canada

${ }^{2}$ Department of Obstetrics and Gynecology, Division of Maternal Fetal Medicine, University of Toronto, Mount Sinai Hospital, Toronto, Canada ${ }^{3}$ Department of Laboratory Medicine and Pathobiology, University of Toronto, Mount Sinai Hospital, Toronto, Canada

\section{Corresponding author:}

Nadine Shehata, Department of Medicine, Division of Hematology, University of Toronto, Mount Sinai Hospital, 6-502-5, 600 University Avenue, Toronto, ON M5G IX5, Canada.

Email: nshehata@mtsinai.on.ca 
Immune thrombocytopenia. ITP is a 'heterogeneous disorder' that induces the development of platelet autoantibodies. ${ }^{14}$ Platelet autoantibodies arise from varied immune mechanisms, such as infection (e.g. Helicobacter pylori), immune dysregulation (e.g. coexistent immune deficiency) and molecular mimicry following infection. ${ }^{14}$ The diagnosis of ITP is considered when the platelet count is consistently below $100 \times 10^{9} / \mathrm{L}^{15}$ In addition to the investigations in Table 1, quantitative Ig levels, direct antiglobulin testing and screening for $H$. pylori can be considered for individuals with ITP suspected of having primary immune deficiency, concurrent autoimmune haemolytic anaemia or recent onset ITP, respectively. ${ }^{7}$ A bone marrow examination is not required for the diagnosis of ITP. ${ }^{2}$

ITP is not infrequently encountered during pregnancy, affecting 2 to $3 / 1000$ pregnancies. ${ }^{5}$ Approximately two-thirds of women have a diagnosis of ITP prior to pregnancy. ${ }^{5}$ Unlike GT, ITP can occur in the first or early second trimester and is one of the more common causes of thrombocytopenia in early pregnancy (Figure 1).

\section{Pregnancy with ITP}

Most women with ITP have mild to moderate thrombocytopenia, and only $30-35 \%$ require any intervention during pregnancy. ${ }^{16,17}$ Most individuals do not have bleeding symptoms. Mild bleeding symptoms, such as easy bruising and purpura, occur in $10 \%$ of individuals, whereas $20 \%$ may experience moderate bleeding, defined as epistaxis, bleeding after trauma and mucous membrane bleeding. ${ }^{16}$ Of women who are symptomatic, a small proportion $(3.4 \%)$ may experience severe bleeding, such as gastrointestinal bleeding, haematuria or deep tissue bleeding. ${ }^{16}$ Overall, ITP is associated with a low risk of maternal

Table I. Investigations for isolated thrombocytopenia in the absence of a family history of bleeding.

Complete blood count and film

Liver enzymes

Thyroid function tests

Vitamin BI2 and folate ${ }^{\mathrm{a}}$

$\mathrm{HCV}, \mathrm{HIV}$ and HBV

Coagulation screen: aPTT and PT

Antinuclear antibody, anticardiolipin antibodies and lupus inhibitor

Medical imaging to assess spleen size

aPTT: activated prothrombin time; PT: prothrombin time.

${ }^{\text {a }}$ Folate deficiency is uncommon in countries with folate fortification. morbidity, and only $10 \%$ of individuals experience exacerbation of their disease postpartum. ${ }^{18}$

\section{Indications for maternal treatment for ITP}

Treatment for ITP during pregnancy is considered in the first or second trimester, if the platelet count is $<20-30 \times 10^{9} / \mathrm{L}$ in the absence of bleeding and if $<50 \times 10^{9} / \mathrm{L}$ in the third trimester, because a platelet count of $50 \times 10^{9} / \mathrm{L}$ or more is adequate for both vaginal delivery and caesarean section. ${ }^{2,16}$

Similar to the treatment for ITP in the nonpregnant individual, the use of corticosteroids and intravenous immune globulin (IVIG) is the treatment option during pregnancy. ${ }^{2}$ A platelet increment in response to corticosteroids occurs after three to seven days. Corticosteroids are associated with a longer duration of response. ${ }^{19}$ Corticosteroids may be associated with a small risk of orofacial clefts during embryogenesis and may aggravate maternal hypertension or diabetes later in pregnancy. ${ }^{20,21}$ The optimum dose of prednisone has not been determined and lower initial doses of prednisone (10-40 mg daily) in comparison to doses used in nonpregnant individuals have been suggested to reduce the adverse effects of prednisone. ${ }^{2,7}$ The lowest effective dose is used during the course of pregnancy.

A rapid increment, within $24-72 \mathrm{~h}$ in platelet count, occurs with IVIG but the increase in platelet count is transient usually lasting only two to three weeks. IVIG is administered at the same dose as in nonpregnant individuals, 1 or $2 \mathrm{~g} / \mathrm{kg}$ over two days. Platelet transfusion is reserved for bleeding patients with platelet counts $<10 \times 10^{9} / \mathrm{L}$, or at delivery if platelet counts are $<30$ to $50 \times 10^{9} / \mathrm{L}$, because transfused platelets are associated with a short duration of response as they are rapidly destroyed.

Second-line treatments for ITP include splenectomy, preferably in the second trimester, ${ }^{22}$ azathioprine, anti-D and cyclosporine. ${ }^{19}$ Rituximab crosses the placenta and may cause neonatal B cell depression if given within six months of delivery. ${ }^{23}$ Vinca alkaloids, cyclophosphamide, danazol and thrombopoietin mimetics should be avoided in pregnancy. ${ }^{19}$

\section{Obstetric management in women with ITP}

The mode of delivery should be determined by obstetrical indications as vaginal delivery has not been associated with increased risk of neonatal bleeding. ${ }^{24,25}$ However, as antiplatelet $\mathrm{IgG}$ antibodies can cross the placenta, resulting in platelet counts $<50 \times 10^{9} / \mathrm{L}$ in 10 to $15 \%$ of

\begin{tabular}{|l|l|}
\hline \multicolumn{2}{|c|}{ Isolated thrombocytopenia } \\
Gestational thrombocytopenia
\end{tabular}

Figure I. Characteristics differentiating gestational thrombocytopenia from immune thrombocytopenia (printed with permission). 
neonates, ${ }^{16}$ fetal blood sampling antepartum, the use of fetal scalp electrodes and performing scalp $\mathrm{pH}$ or lactate in labour, are not advised as they potentially increase risk of fetal bleeding. ${ }^{7}$ In addition, fetal scalp sampling may be inaccurate. ${ }^{26}$ Assisted vaginal delivery should be approached cautiously, considering the risks of bleeding to the neonate as compared to the risks associated with a second-stage caesarean section. ${ }^{27}$ If assisted vaginal delivery is performed, use of forceps is preferable to use of a ventouse cup, as vacuum-assisted deliveries have been associated with an increase in the risk of neonatal intracranial haemorrhage. ${ }^{28}$ The platelet threshold required for neuraxial anaesthesia has not been determined but in practice, most anaesthetists accept a lower limit of platelet count of $50-80 \times 10^{9} /$ L. $^{2}$

\section{Maternal ITP and the neonate}

Although the risk of neonatal thrombocytopenia is low, a cord platelet count should be obtained. If the platelet count is $<50 \times 10^{9} / \mathrm{L}$, the decision to administer intramuscular vitamin $\mathrm{K}$ is balanced with the risk of haemorrhagic disease of the newborn and the efficacy of oral formulations.

Similarly, neonatal intramuscular vaccinations should be delayed until the platelet count has recovered. Although the risk of neonatal intracranial haemorrhage is $<1 \%$, neonates with platelet counts $<50 \times 10^{9} / \mathrm{L}$ should have a cranial ultrasound to exclude its presence. ${ }^{10}$ Fetal/neonatal alloimmune thrombocytopenia should also be considered in neonates with platelet counts $<50 \times 10^{9} / \mathrm{L}$.

The neonatal platelet count usually reaches a nadir three to five days postpartum ${ }^{2,16}$ but may be reduced for months. ${ }^{2}$ The most consistently demonstrated risk factor for neonatal thrombocytopenia is a sibling with a history of thrombocytopenia, though a mother with ITP refractory to splenectomy may also increase risk. ${ }^{16,29,30}$ There is no correlation of neonatal platelet counts with maternal platelet counts ${ }^{5}$ or with the maternal use of betamethasone. ${ }^{31}$ The neonate's platelet count should be monitored until a normal platelet number is reached.

\section{Case I resolution}

This individual has a finding of isolated thrombocytopenia in the late second trimester. All investigations have excluded other possible diagnoses. Although the likely aetiology is GT, ITP cannot be reliably excluded. If the platelet count remains at this level, she may have neuraxial anaesthesia safely and delivery is guided by obstetrical indications. As ITP is still considered an alternate diagnosis, antepartum umbilical cord sampling, intrapartum monitoring of scalp $\mathrm{pH}$ or lactate and assisted vacuum delivery should be avoided. A cord platelet count and serial monitoring of neonatal platelet counts should be undertaken. In addition, intramuscular injections are generally avoided. Maternal and serial neonatal platelet counts are advised postpartum until thrombocytopenia has resolved.

\section{Case 2}

A 31-year-old gravida 1 para 0 at 28 weeks' gestation has a platelet count of $20 \times 10^{9} / \mathrm{L}$, haemoglobin concentration of $79 \mathrm{~g} / \mathrm{L}$ and peripheral blood film demonstrating $10 \%$ schistocytes. Her blood pressure is $140 / 90 \mathrm{mmHg}$, and she has no neurological deficits. Serum creatinine, liver enzymes and urinary protein levels are normal.

Thrombocytopenia associated with microangiopathic haemolytic anaemia, secondary to endothelial damage, and characterized by schistocytes (fragments) on peripheral blood film occurs with several disorders $^{32}$ (Table 2). Laboratory findings, aside from reduction in the haemoglobin concentration and thrombocytopenia, include elevation in lactate dehydrogenase (LDH) concentration, reduction in haptoglobin levels and elevation in indirect bilirubin levels. There is
Table 2. Disorders associated with microangiopathic haemolytic anaemia and thrombocytopenia. ${ }^{32}$

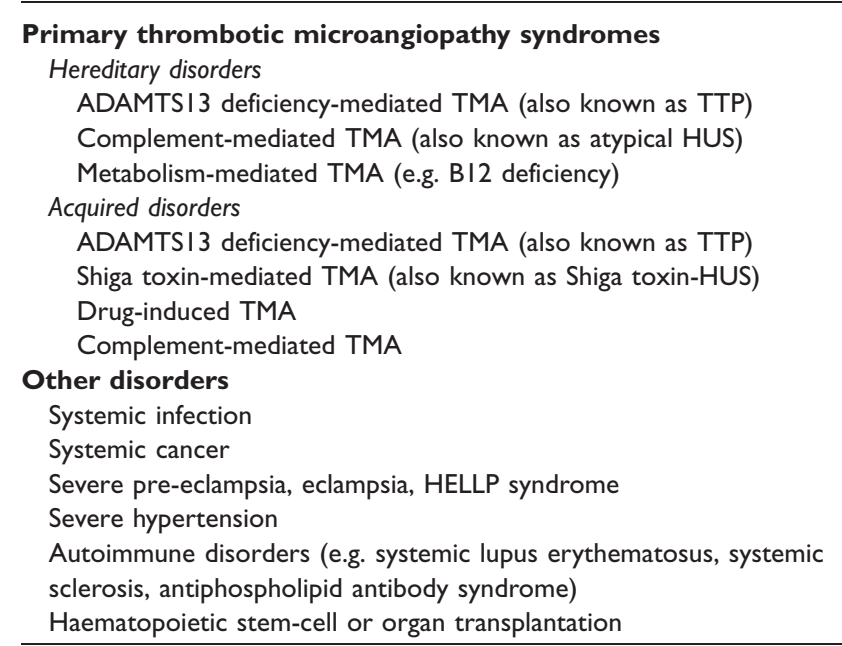

ADAMTSI3: A disintegrin and metalloproteinase with thrombospondin motifs 13; HUS: hemolytic uremic syndrome; TMA: thrombotic microangiopathy; TTP: thrombotic thrombocytopenic purpura.

considerable overlap among the syndromes so that the correct diagnosis may not initially be easily identifiable (Table 3).

\section{Thrombocytopenia associated with systemic disease}

Primary thrombotic microangiopathies. The thrombotic microangiopathies (TMAs) are a group of disorders characterized by microangiopathic haemolytic anaemia, thrombocytopenia and organ injury. ${ }^{32}$ This includes A disintegrin and metalloproteinase with thrombospondin motifs 13 (ADAMTS13)-deficient TMA (thrombotic thrombocytopenic purpura (TTP)) and complement-mediated TMA (atypical haemolytic uremic syndrome). TTP is caused by a deficiency in the von Willebrand factor cleaving protease ADAMTS13 that cleaves multimeric von Willebrand factor. This results in ultralarge von Willebrand factor multimers that lead to platelet thrombi in small vessels. Complement-mediated TMA is due to from over activation of the alternative pathway of complement. ${ }^{32}$

ADAMTS/3-deficient TMA (TTP). TTP occurs in 1 in 25,000 pregnancies. ${ }^{19}$ A marked reduction in ADAMTS13 activity $(<10 \%)$ and evidence of thrombocytopenia and microangiopathic haemolytic anaemia are suggestive of TTP. The presence of anti-ADAMTS13 antibodies in addition to very low levels of ADATMS13 establishes the diagnosis of acquired TTP, whereas the absence of ADAMTS13 antibodies suggests hereditary TTP. A mild reduction in ADAMTS13 levels in the absence of other findings may also occur with normal pregnancy. ${ }^{33}$

Hereditary TTP can occur in any trimester, ${ }^{34}$ but acquired TTP usually manifests in the second and third trimesters. ${ }^{33}$ The platelet nadir with TTP tends to be lower than seen with haemolysis, elevated liver enzymes and low platelets (HELLP) syndrome, with platelet counts $<20 \times 10^{9} /$ L suggesting TTP. ${ }^{35}$ Maternal survival has improved markedly since the implementation of treatment with plasma infusion and plasma exchange, ranging between 88 and $100 \%{ }^{33,34}$ However, fetal survival is approximately $60 \%$, with the majority of fetal loss occurring when TTP occurs in the first or second trimester. ${ }^{33-35}$ The risk of recurrence in subsequent pregnancies is estimated to be $50 \%{ }^{33}$

TTP is a medical emergency. Plasma infusion is used as a replacement for ADAMTS13 for hereditary TTP, while plasma exchange and immune suppressants (e.g. corticosteroids) are used for acquired TTP. 


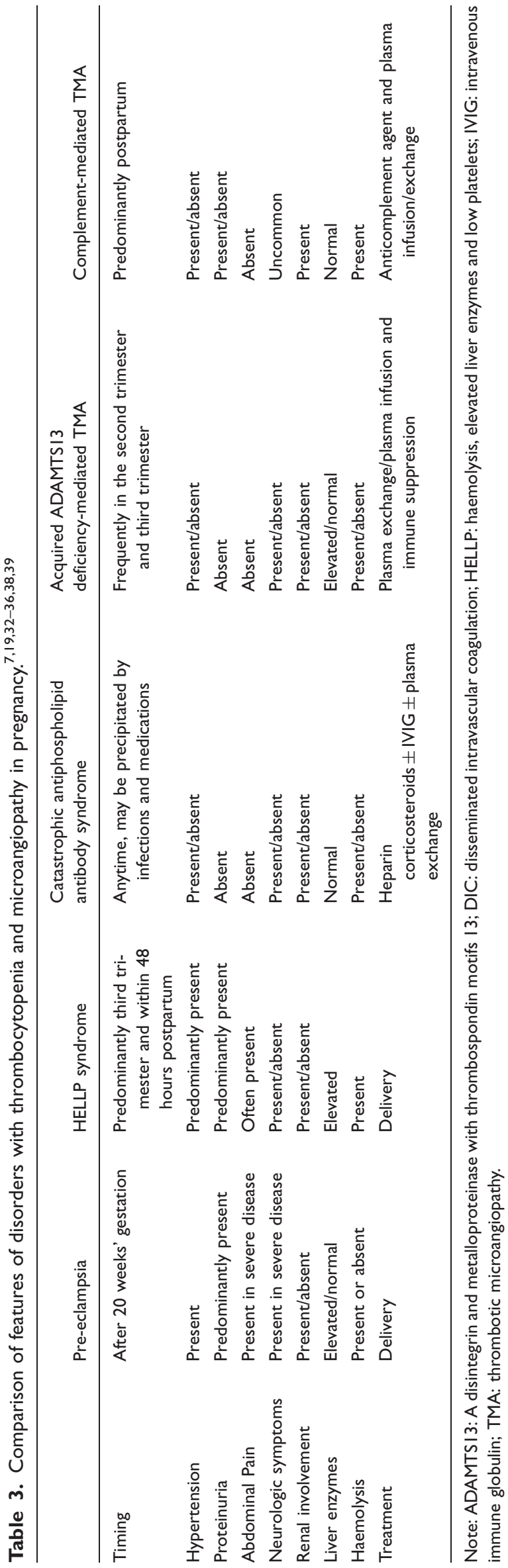

Antenatal use of low-dose aspirin and low-molecular-weight heparin has been recommended in individuals with platelet counts above $50 \times 10^{9} / \mathrm{L}$ but is not considered a standard regimen. ${ }^{35}$ Serial ultrasound scans for fetal growth and well-being should be instituted in the late second and third trimesters, given the risk of intrauterine growth restriction and intrauterine fetal demise.

Complement-associated TMA. Complement-associated TMA predominantly occurs postpartum, and renal involvement is more common. ${ }^{36}$ Since it can be difficult to distinguish from TTP, plasma exchange remains the first-line treatment. If an individual does not respond to plasma exchange, or the ADAMTS13 level is normal, then complement-associated TMA is suspected and a complement inhibitor, such as eculizumab, is instituted. The risk of recurrence of complement-associated TMA in subsequent pregnancies is approximately $20 \%{ }^{36}$

\section{Pre-eclampsia}

Pre-eclampsia affects approximately $6 \%$ of all pregnancies. ${ }^{19}$ Pre-eclampsia is a systemic disorder characterized by new-onset hypertension $(\geq 140 / 90 \mathrm{mmHg}$ ) after 20 weeks' gestational age, with involvement of at least one other system. This may be renal, hepatic, neurological, fetal or haematological. Fifty percent of patients with pre-eclampsia will have platelet counts $<150 \times 10^{9} / \mathrm{L}^{37}$

The HELLP syndrome represents a severe manifestation of preeclampsia. HELLP occurs in approximately 10 to $20 \%$ of women with pre-eclampsia ${ }^{38}$ and is often accompanied by right upper quadrant or epigastric pain, whereas hypertension and proteinuria may be mild or absent. ${ }^{39}$ HELLP predominantly occurs in the third trimester; however, $30 \%$ of women may present within $48 \mathrm{~h}$ postpartum. ${ }^{19}$ The perinatal mortality rate is high, between 7 and $34 \% .{ }^{38}$

Pre-eclampsia and HELLP resolve within days to months after delivery. Corticosteroids have not been shown to improve clinical outcomes. ${ }^{40}$ If HELLP does not improve or deteriorates after three days postpartum, has onset more than seven days postpartum or is accompanied by persistent or worsening neurological or renal dysfunction, an alternative diagnosis such as a primary TMA must be considered. $^{25}$

\section{Disseminated intravascular coagulation}

Disseminated intravascular coagulation (DIC) is a syndrome characterized by a 'maladaptive' activation of coagulation in response to injury. ${ }^{41}$ Pregnancy-specific precipitants of DIC include intrauterine fetal demise, pre-eclampsia, HELLP syndrome ${ }^{41}$ and acute fatty liver of pregnancy. ${ }^{42}$ The characteristics of DIC are prolonged prothrombin time (PT) and activated partial thromboplastin time (aPTT), low platelet counts, low fibrinogen concentration and elevated products of fibrin degradation, e.g. D-dimer. As the PT and aPTT are shortened during pregnancy secondary to increased coagulation factors, a prolongation in the PT or aPTT from baseline may be indicative of DIC, even if the $\mathrm{PT}$ and aPTT are within the normal range. Thus, a change in levels is more important than the absolute result. ${ }^{41}$

The cornerstone of DIC management is to correct the underlying precipitant as DIC will then usually subside. In the absence of haemorrhage, correction of coagulopathies is not warranted. In the presence of bleeding or if emergent delivery is required, platelets are administered if the platelet count is $<50 \times 10^{9} / \mathrm{L}$. Plasma is administered at a dose of 15 to $20 \mathrm{~mL} / \mathrm{kg}$ for coagulopathies, whereas hypofibrinogenemia may be corrected by administering cryoprecipitate or fibrinogen concentrate depending on component availability. Cryoprecipitate at a dose of two pools and fibrinogen concentrate at a dose of $2-4 \mathrm{~g}$ are used to target a fibrinogen level of more than $1.5-2.0 \mathrm{~g} / \mathrm{L} .{ }^{41}$ Red cell transfusion is advised in a haemorrhaging patient to maintain haemodynamic stability. 


\section{Case 2 resolution}

Additional investigations in this individual show an increased LDH level, markedly reduced haptoglobin concentration and elevated indirect bilirubin, consistent with haemolysis. The peripheral blood film suggests microangiopathy. The absence of proteinuria and normal liver enzymes diminishes the likelihood of HELLP, and the normal creatinine concentration excludes the diagnosis of complementmediated TMA. The diagnosis is most consistent with TTP. Because the results of ADAMTS13 testing are not available immediately in most laboratories, plasma exchange should be instituted immediately. If plasma exchange is not available, plasma infusion is used until transfer can be arranged to a centre that offers plasma exchange.

\section{Declaration of conflicting interests}

The author(s) declared no potential conflicts of interest with respect to the research, authorship, and/or publication of this article.

\section{Funding}

The author(s) disclosed receipt of the following financial support for the research, authorship, and/or publication of this article: NS received support from a Canadian Institute of Health Research/Canadian Blood Services New Investigator Award.

\section{Guarantor}

NS.

\section{Contributorship}

MY and NS drafted and revised the manuscript. AKM revised the manuscript.

\section{Patient consent}

The cases are hypothetical.

\section{References}

1. Verdy E, Bessous V, Dreyfus M, et al. Longitudinal analysis of platelet count and volume in normal pregnancy. Thromb Haemost 1997; 77: 806-807.

2. Provan D, Stasi R, Newland AC, et al. International consensus report on the investigation and management of primary immune thrombocytopenia. Blood 2010; 115: 168-186.

3. Boehlen F, Hohlfeld P, Extermann P, et al. Platelet count at term pregnancy: a reappraisal of the threshold. Obstet Gynecol 2000; 95 : 29-33.

4. Burrows RF and Kelton JG. Thrombocytopenia at delivery: a prospective survey of 6715 deliveries. Am J Obstet Gynecol 1990; 162: 731-734.

5. Burrows RF and Kelton JG. Fetal thrombocytopenia and its relation to maternal thrombocytopenia. N Engl J Med 1993; 329: 1463-1466.

6. Win N, Rowley M, Pollard C, et al. Severe gestational (incidental) thrombocytopenia: to treat or not to treat. Hematology 2005; 10 : $69-72$.

7. Gernsheimer T, James JH and Stasi R. How I treat thrombocytopenia in pregnancy. Blood 2013; 121: 38-47.

8. Pierrot-Deseilligny Despujol C, Michel M, Khellaf M, et al. Antiphospholipid antibodies in adults with immune thrombocytopenic purpura. Br J Haematol 2008; 142: 638-643.

9. Miyakis S, Lockshin MD, Atsumi T, et al. International consensus statement on an update of the classification criteria for definite antiphospholipid syndrome (APS). J Thromb Haemost 2006; 4: 295-306.
10. Cuadrado MJ, Mujic F, Muñoz E, et al. Thrombocytopenia in the antiphospholipid syndrome. Ann Rheum Dis 1997; 56: 194-196.

11. Cervera R, Serrano R, Pons-Estel GJ, et al. for the EuroPhospholipid Project Group (European Forum on Antiphospholipid Antibodies) Morbidity and mortality in the antiphospholipid syndrome during a 10-year period: a multicentre prospective study of 1000 patients. Ann Rheum Dis 2015; 74: 1011-1018.

12. Pengo V, Ruffatti A, Legnani $C$, et al. Incidence of a first thromboembolic event in asymptomatic carriers of high-risk antiphospholipid antibody profile: a multicenter prospective study. Blood 2011; 118: 4714-4718.

13. Lippi G and Plebani M. EDTA-dependent pseudothrombocytopenia: further insights and recommendations for prevention of a clinically threatening artifact. Clin Chem Lab Med 2012; 50: $1281-1285$.

14. Cines DB, Bussel JB, Liebman HA, et al. The ITP syndrome: pathogenic and clinical diversity. Blood 2009; 113: 6511-6521.

15. Rodeghiero F, Stasi R, Gernsheimer T, et al. Standardization of terminology, definitions and outcome criteria in immune thrombocytopenic purpura of adults and children: report from an international working group. Blood 2009; 113: 2386-2393.

16. Webert KE, Mittal R, Sigouin C, et al. A retrospective 11-year analysis of obstetric patients with idiopathic thrombocytopenic purpura. Blood 2003; 102: 4306-4311.

17. Veneri D, Franchini M, Raffaelli R, et al. Idiotpathic thrombocytopenic purpura in pregnancy: analysis of 43 consecutive cases followed at a single Italian institution. Ann Hematol 2006; 85: $552-554$.

18. Loustau V, Debouverie O, Canoui-Poitrine F, et al. Effect of pregnancy on the course of immune thrombocytopenia: a retrospective study of 118 pregnancies in 82 women. Br J Haematol 2014; 166: 929-935.

19. Myers B. Diagnosis and management of maternal thrombocytopenia in pregnancy. Br J Haematol 2012; 158: 3-15.

20. Hviid A and Molgaard-Nielsen D. Corticosteroid use during pregnancy and risk of orofacial clefts. CMAJ 2011; 183: 796-804.

21. Park-Wyllie L, Mazzotta P, Pastuszak A, et al. Birth defects after maternal exposure to corticosteroids: prospective cohort study and meta-analysis of epidemiological studies. Teratology 2000; 62: 385-392.

22. Griffiths J, SIa W, Shapiro AM, et al. Laparascopic splenectomy for the treatment of refractory immune thrombocytopenia in pregnancy. J Obstet Gynaecol Can 2005; 27: 771-774.

23. Chakravarty EF, Murray ER, Kelman A, et al. Pregnancy outcomes after maternal exposure to rituximab. Blood 2011; 117: 1499-1506.

24. Cook RL, Miller RC, Katz VL, et al. Immune thrombocytopenic purpura in pregnancy: a reappraisal of management. Obstet Gynecol 1991; 78: 578-583.

25. Ozkan H, Cetinkaya M, Köksal N, et al. Neonatal outcomes of pregnancy complicated by idiopathic thrombocytopenic purpura. J Perinatol 2010; 30: 38-44.

26. Christiaens GC and Helmerhorst FM. Validity of intrapartum diagnosis of fetal thrombocytopenia. Am J Obstet Gynecol 1987; 157: 864-865.

27. Allen VM1, O'Connell CM and Baskett TF. Maternal and perinatal morbidity of caesarean delivery at full cervical dilatation compared with caesarean delivery in the first stage of labour. BJOG 2005; 112: 986-990.

28. Ekéus C, Högberg U and Norman M. Vacuum assisted birth and risk for cerebral complications in term newborn infants: a population-based cohort study. BMC Preg Childbirth 2014; 14: 36.

29. Koyoma S, Tomimatsu T, Kanagawa T, et al. Reliable predictors of neonatal immune thrombocytopenia in pregnant women with idiopathic thrombocytopenic purpura. Am J Hematol 2012; 87: $15-21$. 
30. Kawaguchi K, Matsubara K, Takafuta T, et al. Factors predictive of neonatal thrombocytopenia in pregnant women with immune thrombocytopenia. Int J Hematol 2014; 99: 570-576.

31. Christiaens GL, Nieuwenhuis HK, Von Dem Borne AK, et al. Idiopathic thrombocytopenic purpura in pregnancy: a randomized trial on the effect of antenatal low dose corticosteroids on neonatal platelet count. Br J Obstet Gynaecol 1990; 97: 893-898.

32. George JN and Nester CM. Syndromes of thrombotic microangiopathy. $N$ Engl J Med 2014; 371: 654-666.

33. Scully M, Thomas M, Underwood M, et al. Thrombotic thrombocytopenic purpura and pregnancy: presentation, management and subsequent pregnancy outcomes. Blood 2014; 124: 211-219.

34. Moatti-Cohen M, Garrec C, Wolf M, et al. Unexpected frequency of Upshaw-Schulman syndrome in pregnancy-onset thrombotic thrombocytopenic purpura. Blood 2012; 119: 5888-5897.

35. Scully M, Hunt BJ, Benjamin S, et al. Guidelines on the diagnosis and management of thrombotic thrombocytopenic purpura and other thrombotic microangiopathies. Br J Hematol 2012; 158: 323-335.

36. Fakhouri F, Roumenina L, Provot F, et al. Pregnancy-associated hemolytic uremic syndrome revisited in the era of complement gene mutations. J Am Soc Nephrol 2010; 21: 859-867.
37. Burrows RF, Hunter DJ, Andrew M, et al. A prospective study investigating the mechanism of thrombocytopenia in preeclampsia. Obstet Gynecol 1987; 70: 334-338.

38. Haram K, Svendsen E and Abildgaard U. The HELLP syndrome: clinical issues and management. A review. BMC Preg Childbirth 2009; 9: 8.

39. Martin JN Jr, Rose CH and Briery CM. Understanding and managing HELLP syndrome: the integral role of aggressive glucocorticoids for mother and child. Am J Obstet Gynecol 2006; 195: 914-934.

40. Woudstra DM, Chandra S, Hofmeyr GJ, et al. Corticosteroids for HELLP (hemolysis, elevated liver enzymes, low platelets) syndrome in pregnancy. Cochrane Database Syst Rev 2010; 9: CD008148.

41. Thachil $\mathbf{J}$ and Toh $\mathbf{C H}$. Disseminated intravascular coagulation in obstetric disorders and its acute haematological management. Blood Rev 2009; 23: 167-176.

42. Cervera R, Bucciarelli S and Plasin MA; for the Catastrophic Antiphospholipid Syndrome (CAPS) Registry Project Group (European Forum on Antiphospholipid Antibodies) Catastrophic antiphospholipid syndrome (CAPS): descriptive analysis of a series of 280 patients from the "CAPS Registry". J Autoimmun 2009; 32: $240-245$. 